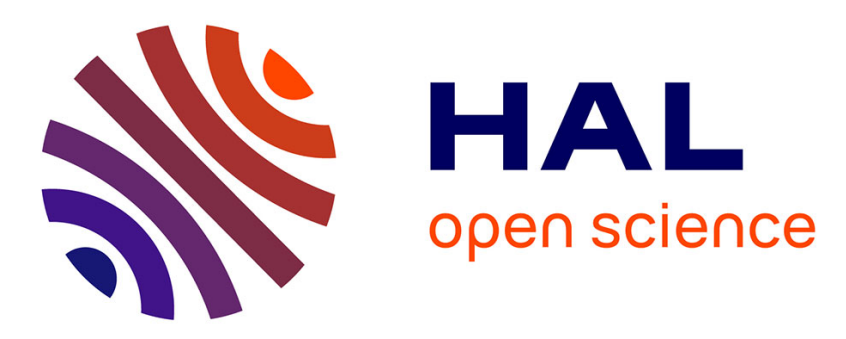

\title{
Impact of spatial filters during sensor selection in a visual p300 brain-computer interface
}

Bertrand Rivet, Hubert Cecotti, Emmanuel Maby, Jérémie Mattout

\section{To cite this version:}

Bertrand Rivet, Hubert Cecotti, Emmanuel Maby, Jérémie Mattout. Impact of spatial filters during sensor selection in a visual p300 brain-computer interface. Brain Topography: a Journal of Cerebral Function and Dynamics, 2012, 25 (1), pp.55-63. 10.1007/s10548-011-0193-y . hal-00660288

\section{HAL Id: hal-00660288 \\ https://hal.science/hal-00660288}

Submitted on 16 Jan 2012

HAL is a multi-disciplinary open access archive for the deposit and dissemination of scientific research documents, whether they are published or not. The documents may come from teaching and research institutions in France or abroad, or from public or private research centers.
L'archive ouverte pluridisciplinaire HAL, est destinée au dépôt et à la diffusion de documents scientifiques de niveau recherche, publiés ou non, émanant des établissements d'enseignement et de recherche français ou étrangers, des laboratoires publics ou privés. 


\title{
Impact of spatial filters during sensor selection in a visual P300 Brain-Computer Interface
}

\author{
B. Rivet ${ }^{1}$, H. Cecotti $^{1}$, E. Maby ${ }^{2}$, J. Mattout ${ }^{2}$ \\ ${ }^{1}$ GIPSA-lab CNRS UMR 5216 \\ Grenoble Universities \\ F-38402 Saint Martin d'Hères, France \\ ${ }^{2}$ INSERM, U821, Lyon, F-69500, France \\ Institut Fédératif des Neurosciences, Lyon, F-69000, France \\ Université Lyon 1, Lyon, F-69000, France
}

\begin{abstract}
A challenge in designing a Brain-Computer Interface (BCI) is the choice of the channels, e.g. the most relevant sensors. Although a setup with many sensors can be more efficient for the detection of Event-Related Potential (ERP) like the P300, it is relevant to consider only a low number of sensors for a commercial or clinical BCI application. Indeed, a reduced number of sensors can naturally increase the user comfort by reducing the time required for the installation of the EEG (electroencephalogram) cap and can decrease the price of the device. In this study, the influence of spatial filtering during the process of sensor selection is addressed. Three sensor selection approaches based on a recursive backward elimination are compared. Two of them maximize the Signal to Signal-plus-Noise Ratio (SSNR) for the different sensor subsets while the third one maximizes the differences between the averaged P300 waveform and the non P300 waveform. We show that the locations of the most relevant sensors subsets for the detection of the P300 are highly dependent on the use of spatial filtering. Applied on data from 20 healthy subjects, this study proves that subsets obtained where sensors are suppressed in relation to their individual SSNR are less efficient than when sensors are suppressed in relation to their contribution once the different selected sensors are combined for enhancing the signal. In other words, it highlights the difference between estimating the P300 projection on the scalp and evaluating the more efficient sensor subsets for a P300-BCI. Finally, this study explores the issue of channel commonality across subjects. The results support the conclusion that spatial filters during sensor selection procedure allow selecting better sensors for a visual P300 Brain-Computer Interface.
\end{abstract}

Keywords: Brain-Computer Interface, P300, EEG, BLDA, Sensor selection 
Impact of spatial filters during sensor selection in a visual P300 Brain-Computer Interface2

\section{Introduction}

A Brain-computer interface (BCI) enables a user to communicate through the direct and real-time measurements of brain activity [1]. For patients suffering from severe motor disabilities, e.g. locked-in patients, a BCI may often represent the only mean of communication between the patients and their family. BCIs may actually provide the only communication pathway for patients who are unable to communicate via conventional means [2]. Although a remaining challenge is to get an efficient working BCI, other challenges aim at reducing the cost of a BCI and to bring BCI technology to mass market, to healthy persons for new BCI applications [3, 4]. This necessary step can require the reduction of the number of electrodes and the adaptation of their locations for each user [5]. Indeed, reducing the number of sensors yields more comfort for the user, decreases installation time duration and may substantially reduce the financial cost of the BCI setup since the cost of an EEG cap and an amplifier vary in relation to the number of channels. Besides, the reduction of the number of sensors can also reduce the power consumption for wireless EEG caps [6]. This point is relevant for BCI applications like video games or for other applications where the number of sensors is limited. Finally sensor selection may facilitate the classifier work by selecting a reduced and more relevant set of input features.

Several strategies are possible for selecting a subset of sensors. For instance, one can select sensors based on prior knowledge from neuroscience studies that can provide the source of the potentials. In such a case, the subset is fixed and may jeopardize the performance in some subjects as the optimal sensor subset is subject-dependent [7]. To obtain optimal performance, it is mandatory to clearly identify subject-specific optimal sensor subsets. For a given set of $N$ sensors, there are $2^{N}-1$ different possible subsets, without the empty subset. To find the optimal subset, three main searching 
Impact of spatial filters during sensor selection in a visual P300 Brain-Computer Interface3 approaches can be considered: exhaustive, random or sequential search. The exhaustive search is usually not possible: the search space is often exponentially prohibitive. The sequential and random search does not guarantee optimality as it gives up completeness. However, it is easily implementable and can provide suboptimal solutions given some evaluation criterion. Several variations are described in the literature like the greedy hillclimbing approach, forward selection, backward elimination or bi-directional selection. For instance, recursive feature elimination was used for sensor selection in BCI based on motor imagery $[8,9]$. In this paper, we consider a recursive backward elimination strategy to determine which sensor should be removed in the widely used P300 speller paradigm [10]. The impact of spatial filtering during the selection of the more relevant sensor subsets is then evaluated with the P300 speller accuracy. Moreover an analysis of commonality of selected channels across subjects is studied. The remainder of the paper is organized as follows: the sensor selection strategy and the different methods are described in the second section; the materials and the experimental protocol are presented in the third section; and finally, results are compared and discussed in the last section.

\section{Methods}

\subsection{Data modeling: $x D A W N$ framework}

The proposed method to select adaptively a relevant subset of sensors is intimately related to the special structure shared by the data due to the oddball paradigm used in a visual P300 BCI. Indeed, the xDAWN framework [11] provides an algebraic model of the recorded signals $X \in \mathbb{R}^{N_{t} \times N_{s}}$ (where $N_{t}$ is the number of time samples in the recorded EEG signal and $N_{s}$ the number of sensors) by considering that $X$ is composed of three additive terms: (i) the P300 responses $A_{1}$ related to target stimuli, (ii) responses 
Impact of spatial filters during sensor selection in a visual P300 Brain-Computer Interface4 $A_{2}$ common to every stimuli, and (iii) the residual noise $H$. As a consequence, one can write $X$ as

$$
X=D_{1} A_{1}+D_{2} A_{2}+H
$$

where $A_{1} \in \mathbb{R}^{N_{1} \times N_{s}}$ and $A_{2} \in \mathbb{R}^{N_{2} \times N_{s}}$ are the patterns representing one target evoked potential (i.e. the P300 response) whose length is $N_{1}$ samples and one common evoked potential whose length is $N_{2}$ samples, respectively. $D_{1} \in \mathbb{R}^{N_{t} \times N_{1}}$ (resp. $D_{2} \in \mathbb{R}^{N_{t} \times N_{2}}$ ) is a Toeplitz matrix whose first column entries are set to zero except for those that correspond to a target (resp. every) onset which are equal to one. $D_{1} A_{1}$ and $D_{2} A_{2}$ thus represent the superposition of all targets and common responses, respectively. This parametrization takes into account the possible overlapping between target potentials and/or between common potentials and it allows to estimate these responses in the least mean square sense by

$$
\left[\begin{array}{c}
\hat{A}_{1} \\
\hat{A}_{2}
\end{array}\right]=\left(\left[D_{1} ; D_{2}\right]^{T}\left[D_{1} ; D_{2}\right]\right)^{-1}\left[D_{1} ; D_{2}\right]^{T} X
$$

where $\left[D_{1} ; D_{2}\right]$ is a matrix of size $N_{t} \times\left(N_{1}+N_{2}\right)$ obtained by concatenation of $D_{1}$ and $D_{2}$. xDAWN model (1) and estimation (2) are useful to define the Signal to Signal plus Noise Ratio (SSNR) of the $i$-th sensor by

$$
\rho\left(\mathbf{e}_{i}, X\right)=\frac{\mathbf{e}_{i}^{T} \hat{A}_{1}^{T} D_{1}^{T} D_{1} \hat{A}_{1} \mathbf{e}_{i}}{\mathbf{e}_{i}^{T} X^{T} X \mathbf{e}_{i}},
$$

where $\mathbf{e}_{i} \in \mathbb{R}^{N_{s}}$ is a zero vector but the $i$-th element equal to 1 . $Y \mathbf{e}_{i}$ is thus the $i$-th column of a matrix $Y$.

The purpose of applying spatial filter $\mathbf{u} \in \mathbb{R}^{N_{s}}$ is to create a virtual sensor so that the resulting signal $X \mathbf{u}$ provides a larger $\operatorname{SSNR} \rho(\mathbf{u}, X)$ than $\operatorname{SSNR}$ achieved by actual sensors $\max _{i} \rho\left(\mathbf{e}_{i}, X\right)$. xDAWN framework [11] also provides a way to estimate $N_{f}$ spatial filters $\mathbf{u}_{i}, i \in\left\{1, \cdots, N_{f}\right\}$, that maximize the SSNR (3). 
Impact of spatial filters during sensor selection in a visual P300 Brain-Computer Interface5

\subsection{Sensor selection}

The chosen method for adaptively selecting a relevant subset of sensors is based on a recursive backward elimination. Starting with all sensors, it consists in alternatively testing each sensor for its significance and in removing the least relevant one at each iteration step. An irrelevant sensor is a sensor whose removal barely impairs the performance or selection criterion. In this work, we eliminate two sensors at a time, leaving us with the most significant remaining subset. Elimination goes on until every sensor has been eliminated. At the end of the selection process, sensors can be ranked according to their revealed significance. A relevant sensor will be eliminated at the end of the iterative process while a useless one will be eliminated along the very first iterations.

The cost function that determines the relevance of a sensor subset is based on the evaluation of the SSNR. The cost function of the sensor subset $\mathcal{I}$ can be estimated as a function of the individual $\operatorname{SSNR} \rho\left(\mathbf{e}_{i}, X\right)$ of each actual sensor

$$
s_{a}(\mathcal{I})=\sum_{i \in \mathcal{I}} \rho\left(\mathbf{e}_{i}, X\right)
$$

This solution is denoted $A S$ as actual sensor. Moreover, the SSNR can also be estimated after some spatial filtering. In this case, the score function is estimated globally as a function of the grouped SSNR from the virtual sensors

$$
s_{v}(\mathcal{I})=\sum_{k=1}^{N_{f}} \rho\left(\mathbf{u}_{k}, X E_{\mathcal{I}}\right)
$$

where $E_{\mathcal{I}}$ is the matrix whose columns are the concatenation of vectors $\mathbf{e}_{i}$ with $i \in \mathcal{I}$. This technique is referred as $V S$ as virtual sensor.

As a reference, the score function based on the largest difference between the averaged P300 trials waveform and the averaged non P300 trials waveform is finally considered and this method is denoted (Ref). In this case the score function of the 
Impact of spatial filters during sensor selection in a visual P300 Brain-Computer Interface6

$A S$ :

SSNR

$V S: \quad$ Spatial Filters $(\mathrm{SF}) \quad+$ SSNR

Ref: Largest difference between P300 and Non P300 trials

Table 1. The three criteria for backward selecting the most relevant sensors.

sensor subset $\mathcal{I}$ is estimated as

$$
s_{r}(\mathcal{I})=\sum_{i \in \mathcal{I}}\left(\mathbf{x}_{i}^{P 300}-\mathbf{x}_{i}^{N P 300}\right)^{T}\left(\mathbf{x}_{i}^{P 300}-\mathbf{x}_{i}^{N P 300}\right),
$$

where $\mathbf{x}_{i}^{P 300}$ (resp. $\mathbf{x}_{i}^{N P 300}$ ) is the vector of the averaged P300 (resp. non P300) trials waveform at channel $i$.

Finally, the backward elimination procedure is summarized in Algorithm 1 where the considered score functions are presented in Table 1.

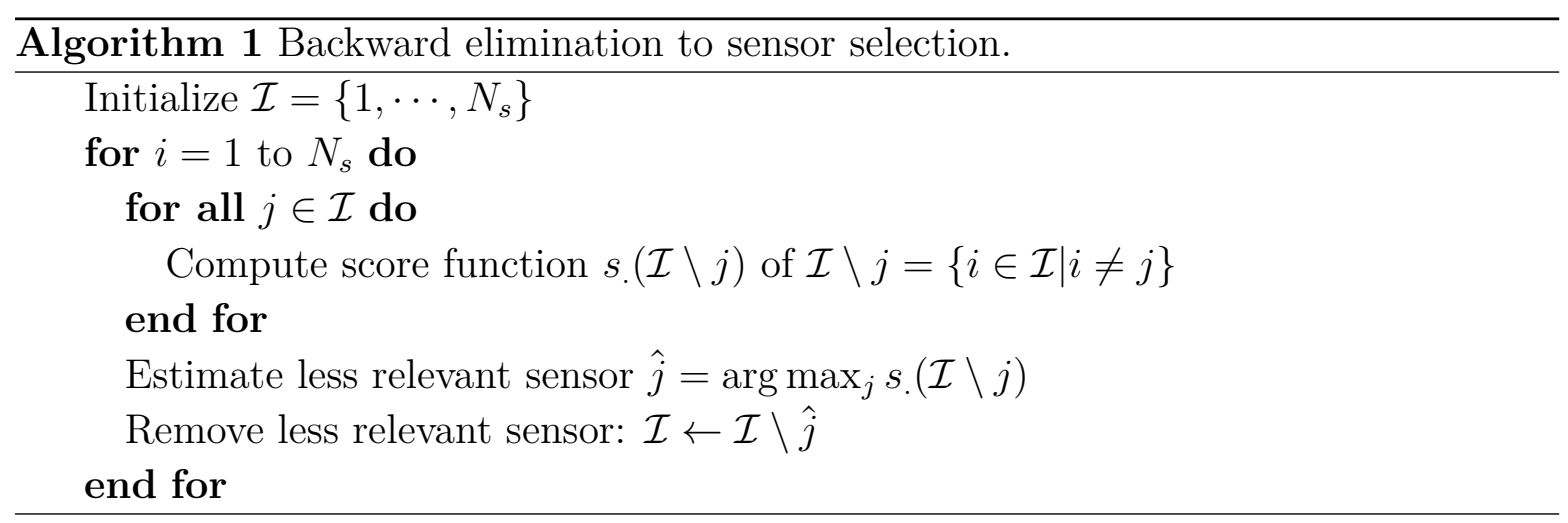

The objectives of the experiments are to evaluate and to compare the impact of the spatial filters during the selection process, i.e. the difference between method $A S$ which eliminates the individual less relevant sensors in relation to their individual SSNR $\rho\left(\mathbf{e}_{i}, X\right)$, and method $V S$ that selects the most relevant sensors in relation to their grouped SSNR $\rho\left(\mathbf{u}, X E_{\mathcal{I}}\right)$.

\section{Materials}

The EEG signal was recorded on 20 healthy subjects (mean age $=26, \mathrm{sd}=5.7 ; 7$ females) with the OpenViBE framework [12]. Each subject filled a consent form prior to the 
Impact of spatial filters during sensor selection in a visual P300 Brain-Computer Interface7 experiment. Subjects were wearing a standard EEG cap with 32 electrodes subsampled from the 10-10 system [13]. The EEG activity was recorded continuously from 32 active electrodes (actiCap, Brain Products GmbH, Munich). The electrodes for the ground and the reference were placed on the forehead and the nose, respectively.

For the evaluation of the spatial filters impact during sensor selection, two sessions were considered. The P300 speller was presented to the subjects as described in [14], with a square matrix of 36 symbols, where one symbol is a letter or a digit. The first session was dedicated to the estimation of different subsets of sensors and for training the classifier. The second session was for testing the P300 speller. In both sessions, the number of repetitions was fixed to 10, i.e. each symbol is spelt 10 consecutive times. The first and second sessions contained 50 and 60 symbols, respectively. The Interval Stimulus Interval (ISI) was 170ms for the training session. For the test session, four groups of 15 characters had an ISI of 110, 150, 190 and 230ms, respectively $\ddagger$. The average ISI in the test session was like the training session, i.e. $170 \mathrm{~ms}$.

The EEG signal was sampled at $100 \mathrm{~Hz}$ and was bandpass filtered between $1 \mathrm{~Hz}$ and $12.5 \mathrm{~Hz}$ with a Butterworth filter (order=4). Finally the features vector is obtained by a downsampling to $25 \mathrm{~Hz}$ for speeding up the computation time and reducing the number of features. Such decimation parameter has been successfully applied in other studies about the P300 speller $[15,16]$. For each sensor and for each trial, the signals were normalized so that they had a zero mean and a standard deviation equal to one. A Bayesian linear discriminant analysis (BLDA) classifier is considered for the detection of the P300 $[7,17]$.

$\ddagger$ Note that in this study, the different ISI are not exploited as a parameter. 


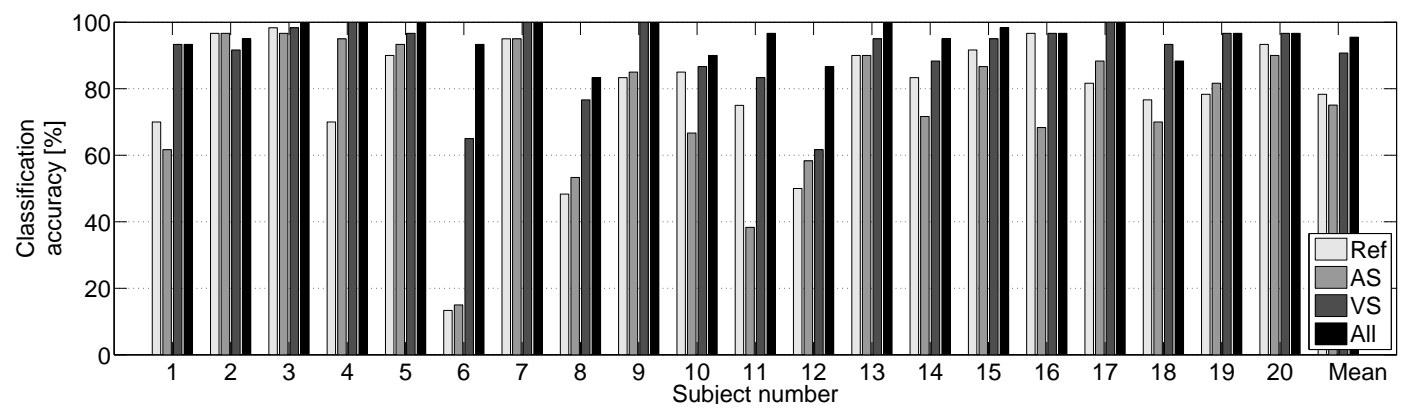

Figure 1. Impact of the sensor subsets on the recognition rate of the P300 speller.

\section{Results}

The P300 speller was evaluated with different sensor subsets as defined in the previous section. For each selected sensor subset, the signal was first enhanced by using spatial filters before the classification stage performed by a BLDA for the detection of the P300. For the classification, a sequence of $0.6 \mathrm{~s}$ from a visual stimulus has been considered for each trial. Therefore, the P300 response is expected to be centered on the chosen time sequence. For each subject, the number of P300 trials and non P300 trials are 1200 and 6000 , respectively, for the test session. Based on a previous study [18], we only consider a subset of four selected sensors by the different methods ( $A S, V S$ and Ref) since it has been shown that four sensors is a good tradeoff between the number of sensors and the classification accuracy. The impact of spatial filters during the selection process is firstly assessed (Section 4.1). The commonality of channels across subjects is then studied (Section 4.2).

\subsection{Impact of spatial filters during sensor selection}

Figure 1 presents the recognition rate of the P300 speller, with 10 repetitions, for the subsets of four sensors obtained with the three methods (AS,VS, Ref), and for the whole set of 32 sensors (All). The average recognition rate is $80.4 \%, 76.4 \%, 90.3 \%$ and 95.8\% for the subsets obtained with Ref, AS, VS and the whole set (All), respectively. 
Impact of spatial filters during sensor selection in a visual P300 Brain-Computer Interface9 The performance of the P300 speller is always better (excepted for subject 2) with the four sensors selected with method $V S$ (i.e. with spatial filters) compared to $A S$ or to Ref. Two-tailed t-tests (5\% significance level) indicate that the method $V S$ provides better sensors than $A S\left(t_{(19)}=4.42, s d=8.4, p<5 e^{-4}\right)$ and than $\operatorname{Ref}\left(t_{(19)}=4.10\right.$, $\left.s d=6.49, p<1 e^{-3}\right)$. A pairwise two-tailed t-test has also been used to compare the speller accuracy with sensors obtained with $V S$ and the whole set. As expected, the result of the t-test indicates a rejection of the null hypothesis at the $5 \%$ significance level. It shows that the whole set of 32 sensors (All) provides a better performance than the 4 sensors obtained with $V S\left(t_{(19)}=2.62, s d=5.36, p<2 e^{-2}\right)$.

The position of the best sensors selected with the methods $A S$ and $V S$ is depicted for every subject in Figure 2. The grey color represents the sensor relevance, i.e. a dark grey means the location is relevant. For both methods, the sensor subsets are not homogeneously located across subjects, highlighting the variability across subjects. In a general way, $V S$ provides sparser sensors than $A S$. Nevertheless, as depicted in Figure 3, the grand average of the evoked response obtained after the spatial filtering step of the four sensors selected by $A S$ and $V S$ show that the $V S$ method provides a better defined evoked response, i.e. a less large P300 wave, than the $A S$ method. Note that the N200 component is also visible before the P300 peak for both $A S$ and $V S$.

Figure 4 (resp. Figure 5) depicts the averaged P300 and non P300 waves across all subjects (resp. for Subject 20) and all sessions. This latter subject is a good example to provide evidence that electrodes with a relative low amplitude can be relevant in a sensor selection procedure. Indeed, one may directly point out the projection of the P300 over $P_{Z}$ as the amplitude of the P300 is higher than with other sensors. However, this electrode was not selected in the best sensor subsets as presented in Figure 1, (S20 (AS) and S20 (VS)). Instead, electrodes closer to the bilateral occipito-temporal parts 
Impact of spatial filters during sensor selection in a visual P300 Brain-Computer Interface10
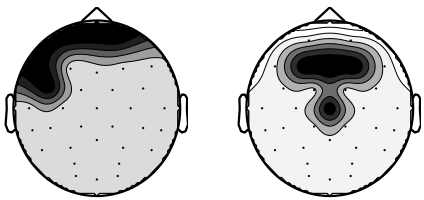

$\mathrm{S} 1(A S)$

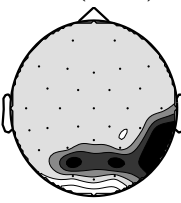

$\mathrm{S} 5(A S)$

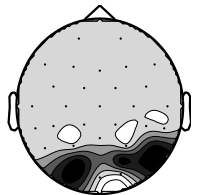

S9 $(A S)$

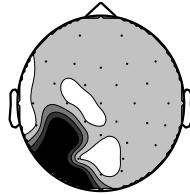

S13 $(A S)$

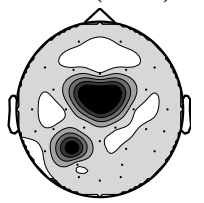

$\mathrm{S} 17(A S)$
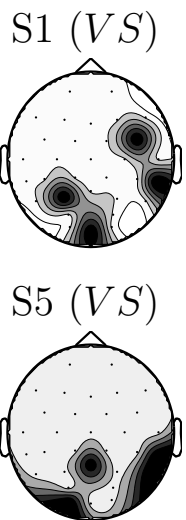

$\mathrm{S} 5(V S)$
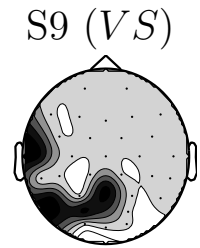

$\mathrm{S} 13(V S)$

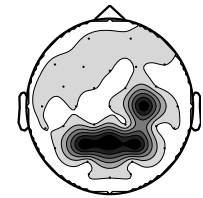

S17 (VS)
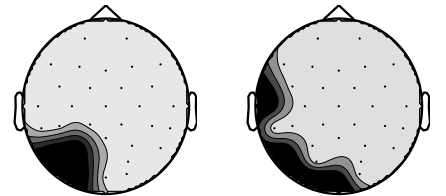

$\mathrm{S} 2(A S)$

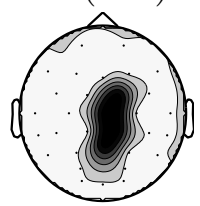

$\mathrm{S} 2(V S)$
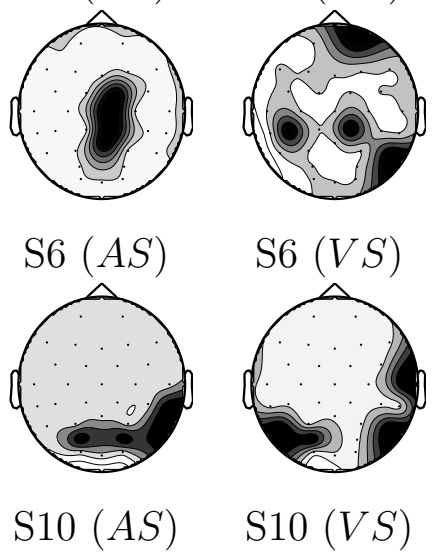

$\mathrm{S} 10(V S)$
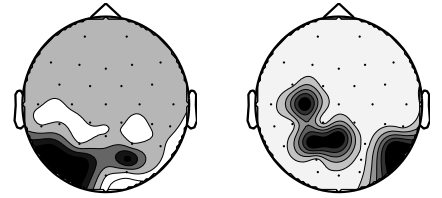

$\mathrm{S} 14(A S)$

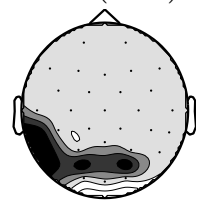

$\mathrm{S} 14(V S)$

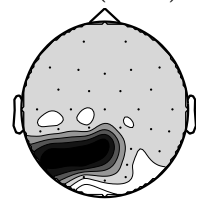

S18 $(A S)$

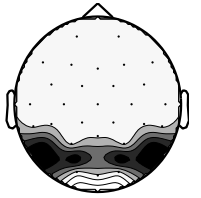

S3 $(A S)$

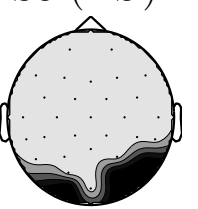

$\mathrm{S} 7(A S)$
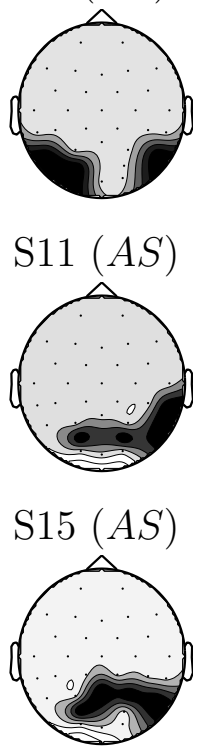

$\mathrm{S} 19(A S)$

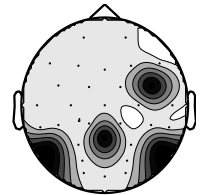

$\mathrm{S} 3(V S)$

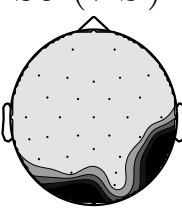

$\mathrm{S} 7(V S)$

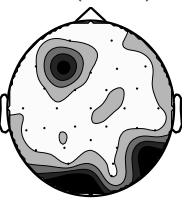

$\mathrm{S} 11(V S)$

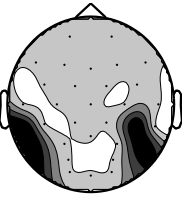

S15 (VS)

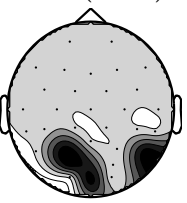

S19 (VS)

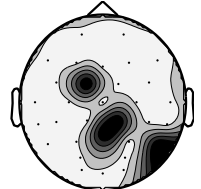

$\mathrm{S} 4(A S)$

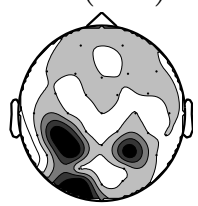

S8 $(A S)$

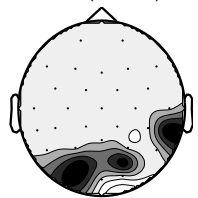

$\mathrm{S} 12(A S)$

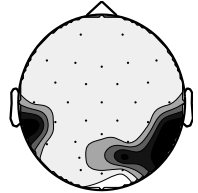

S16 $(A S)$

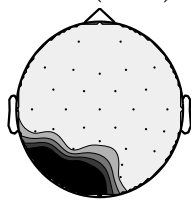

S20 $(A S)$

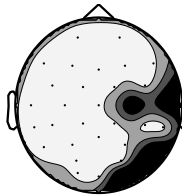

$\mathrm{S} 4(V S)$

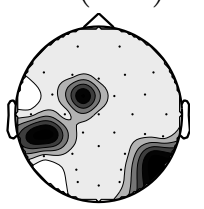

$\mathrm{S} 8(V S)$

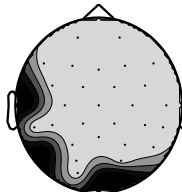

$\mathrm{S} 12(V S)$

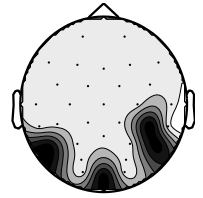

S16 (VS)

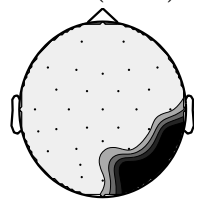

S20 (VS)

Figure 2. Position of the 4 best sensors for the criteria AS and VS. A dark/light grey represents a relevant/non relevant sensor location.

were selected.

\subsection{Commonality of channels across subjects}

In Figure 6, an analysis of the differences and similarities of selected sensors for each subject is provided. The mean, the standard deviation (S.D.) and the kurtosis across the 20 subjects with four selected sensors are depicted in Fig. 6 in the column (a), (b) and (c) respectively. A dark (resp. light) gray level denotes a high (resp. low) rank. The rank represents the iteration during the backward elimination when the sensors 


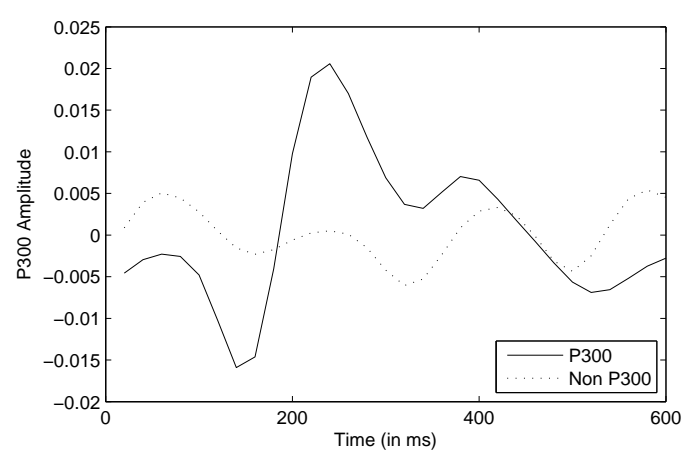

(a) $\mathrm{AS}$

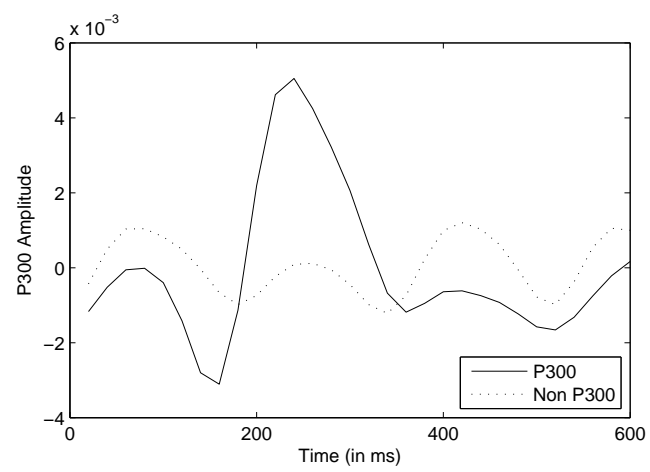

(b) VS

Figure 3. Averaged P300 and non P300 trials after spatial filtering of 4 sensors selected with $A S$ and $V S$, during $600 \mathrm{~ms}$.

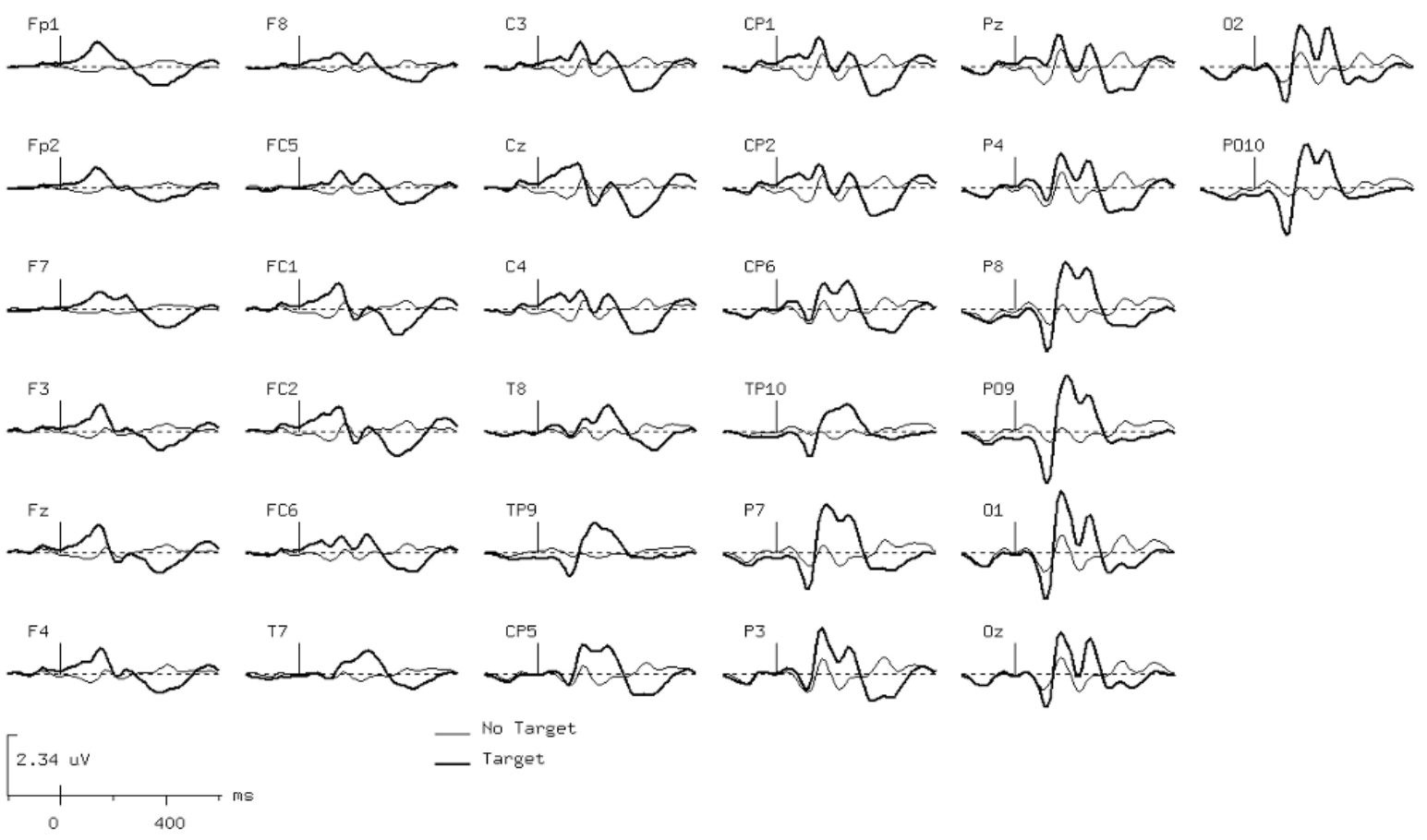

Figure 4. Averaged ERP on the target and non-target across the different electrodes. The bold and thin lines represent the ERP on the target and non target, respectively.

has been removed. A high rank means an important sensor, i.e. a sensor to keep in a reduced subset. The average sensor selection seems quite similar between the methods $A S$ and $V S$ : mainly in the occipital and parietal areas. However, it is challenging to extract some common sensors that may be useful for every subject. The S.D. is higher in the occipital and parietal areas, suggesting that the order of these sensors can vary 


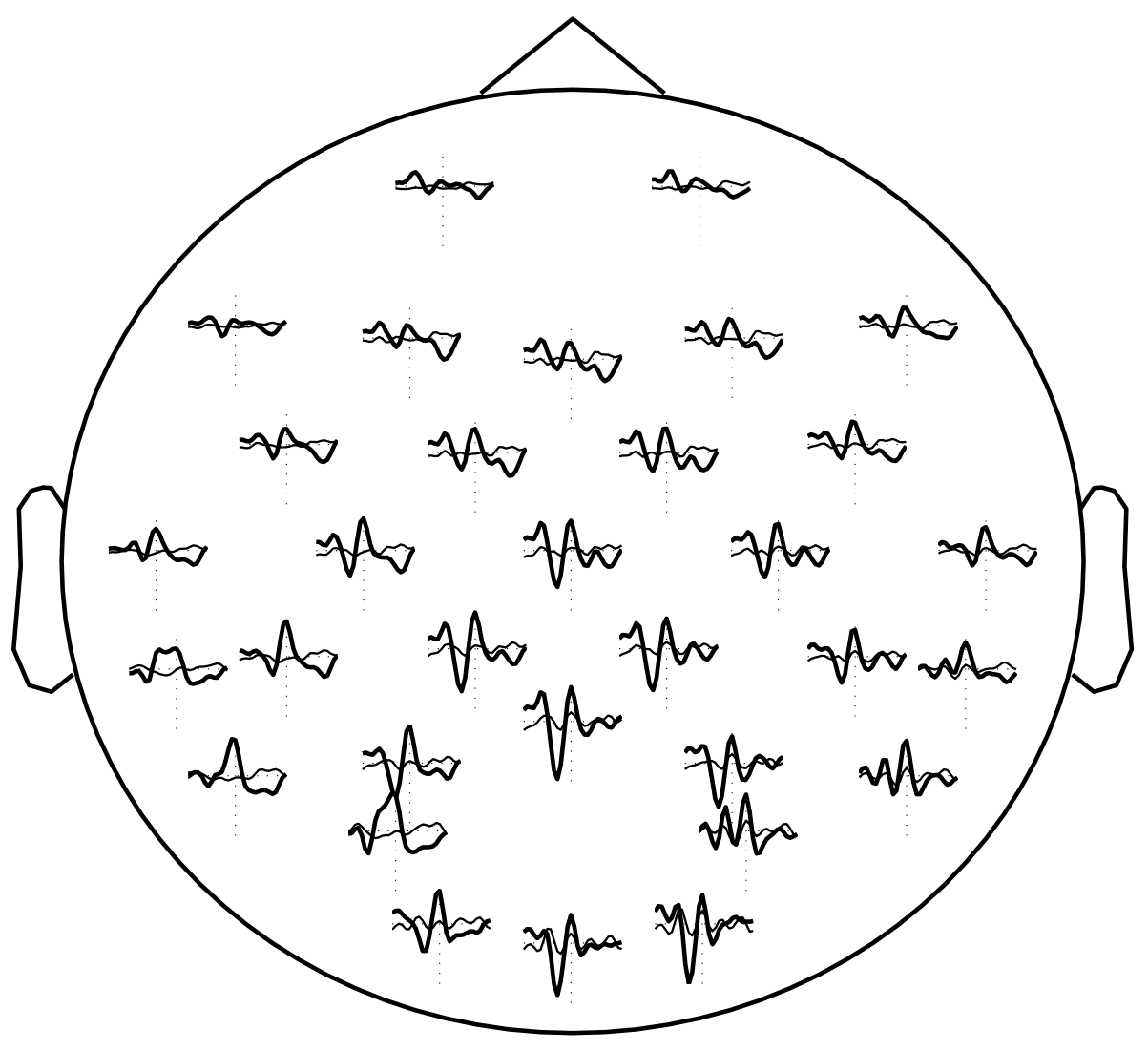

Figure 5. Averaged ERP on the target and non-target across the different electrodes for Subject 20, during $600 \mathrm{~ms}$. The vertical dash line corresponds to $300 \mathrm{~ms}$ after a visual stimulus. The bold/thin line represents the averaged P300/non P300 response.

a lot while keeping a high importance in the sensor selection. Finally, the figures of the last column aim at depicting the sensors that could be relevant for few subjects. The kurtosis of the sensor rank suggests that although the majority of the relevant sensors are in the occipital and parietal area, some sensors in the frontal area remain important for some subjects to enhance the P300.

Even if this last observation proves the necessity to customize the sensor location for each subject so that the performance are maximized, we compare two criteria to select a common subset of four sensors for all the subjects (Table 2 and Figure 7). The first one (denoted 'Voting') is simply obtained by a voting procedure: the sensors are ranked in relation to their frequency of appearance in the four best sensors for the 20 
Impact of spatial filters during sensor selection in a visual P300 Brain-Computer Interface13

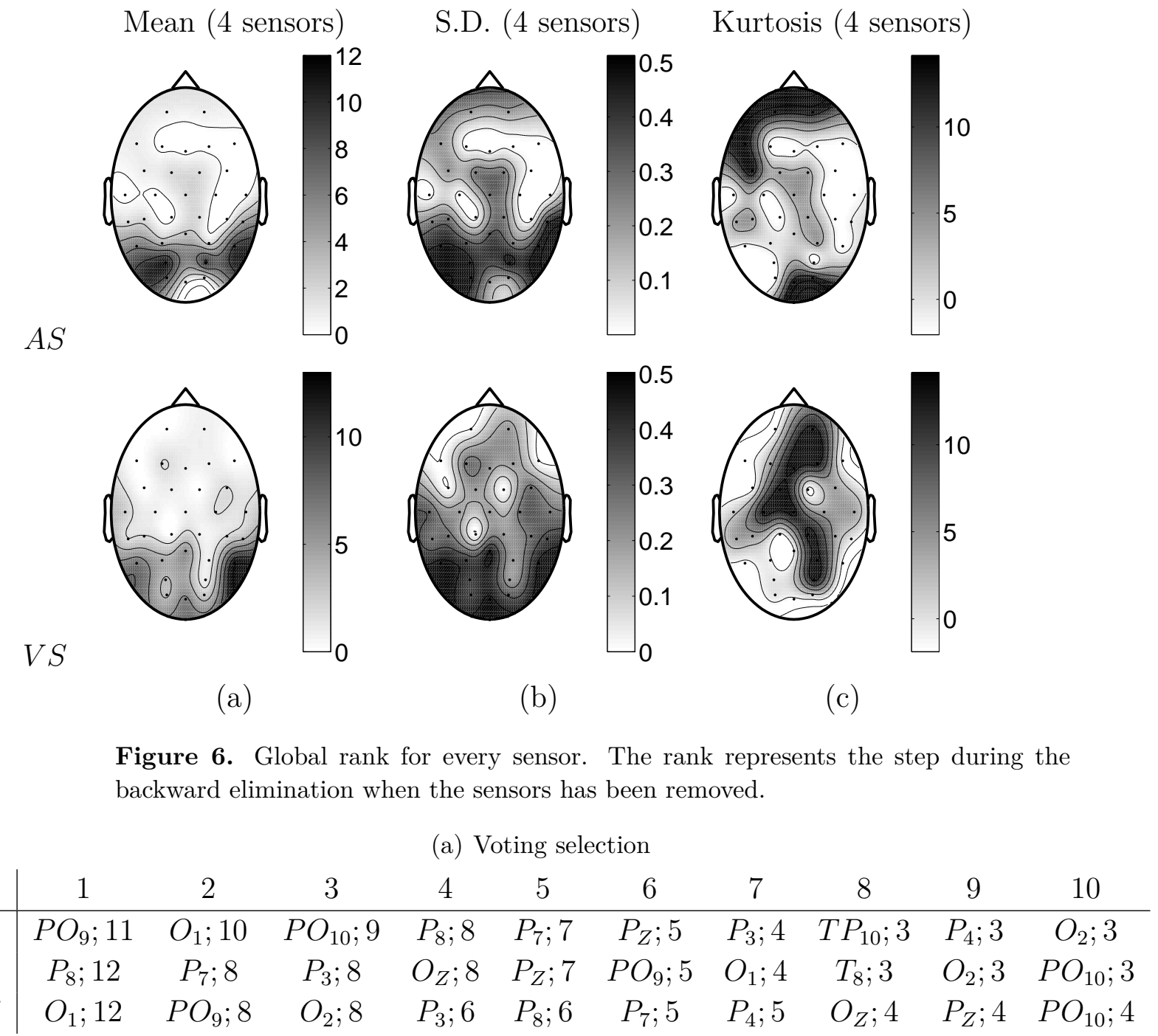

(b) Joint selection

\begin{tabular}{c|cccccccccc} 
Top & 1 & 2 & 3 & 4 & 5 & 6 & 7 & 8 & 9 & 10 \\
\hline$A S$ & $P_{8}$ & $P_{7}$ & $P O_{9}$ & $O_{1}$ & $P_{3}$ & $P O_{10}$ & $O_{2}$ & $P_{4}$ & $O_{z}$ & $P_{z}$ \\
$V S$ & $P O_{9}$ & $P_{3}$ & $C_{Z}$ & $P_{8}$ & $O_{Z}$ & $T_{8}$ & $F_{3}$ & $O_{1}$ & $P_{4}$ & $P_{Z}$ \\
$R e f$ & $O_{1}$ & $P_{8}$ & $P_{3}$ & $P O_{9}$ & $P_{7}$ & $O_{2}$ & $P_{Z}$ & $P_{4}$ & $P O_{10}$ & $O_{Z}$
\end{tabular}

Table 2. Top 10 sensors for $A S$ and $V S$ across 20 subjects selecting by a voting procedure (a) and by a joint selection (b). Each cell represents the electrode position in the international extended 10-10 system. For the voting procedure (a), the frequency this electrode has been selected as one of the four best sensors across the 20 subjects is also indicated.

subjects. The second procedure (denoted 'Joint') refers to the backward elimination procedure (Algorithm 1) where the subject specific score function $s .(\mathcal{I} \backslash j)$ is replaced by the average of the 20 individual score functions related to each subject. 


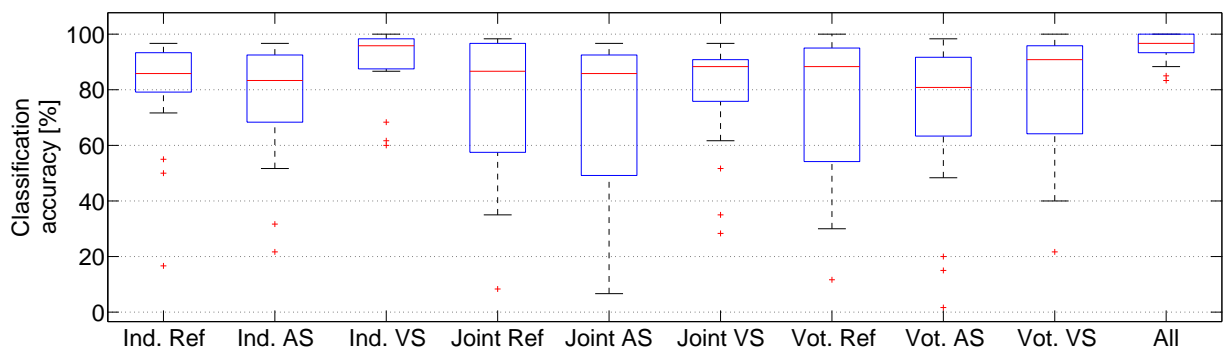

Figure 7. Comparison of classification accuracy achieved for all the 20 subjects by several sensor selection procedures (Ref, $A S$ and $V S$ ). 'Ind.', 'Joint' and 'Vot.' refer to a subject specific selection, to the joint selection and to the voting selection, respectively. All refers to the classification accuracy achieved with all the 32 sensors. On each box, the central mark is the median, the edges of the box are the 25th and 75th percentiles, the whiskers extend to the most extreme data points not considered outliers, and outliers are plotted individually.

As already mentioned, there is a large diversity across subjects about the location of the most relevant sensors. Indeed, the largest value of the frequency of appearance among the four most relevant sensors is only 11 or 12 depending of the selection criterion: this means that this sensor is only common to about half of the subjects. It is also worth noting that the voting and joint selection procedures provide quite different selected sensors. Actually, for the $V S$ method, if $P_{8}$ and $P_{3}$ are among the four selected sensors for both voting and joint selection methods, $\mathrm{PO}_{9}$ is only ranked as the sixth most relevant method by the voting selection whereas it is the most relevant sensor for the joint selection. Even more surprising, $C_{Z}$ (resp. $P_{7}$ ) is in the four most relevant sensor for the joint (resp. voting) method while it is not among the 10 most relevant sensors for the voting (resp. joint) selection. Furthermore, as shown by Figure 7, among the joint and voting selection, the joint and voting selections with virtual sensor (Joint VS and Vot. VS) provide the best classification accuracy. Indeed, with these four sensors common to all the 20 subjects selected by Joint VS (resp. Vot. VS), the classification accuracy is larger than $60 \%$ for 17 (resp. 16) of them and 16 (resp. 14) subjects achieved even more than $75 \%$ of classification accuracy. Moreover eleven subjects achieved an 
Impact of spatial filters during sensor selection in a visual P300 Brain-Computer Interface15 impressive classification accuracy larger than $90 \%$ with only four sensors selected by Vot. VS. Finally, this latter result highlights one more time the fact that using spatial filters during the sensor selection is essential to provide accurate subsets of channels since classification accuracy for both Joint $V S$ and $V$ ot. $V S$ is larger than with $A S$ or Ref methods.

\section{Conclusion and discussion}

Three ways for the analysis of the best sensor location for P300 detection have been proposed. In every solution, the method is based on a recursive backward elimination. In the first case, sensors are suppressed in relation to their individual SSNR. In the second case, sensors are suppressed in relation to their contribution once the different selected sensors are combined for enhancing the signal. In the third solution, sensors are suppressed in relation to the largest differences between P300 and Non P300 trials waveforms. The results indicate that the sensors, which are contained in the best sensor subset are different than the sensors that provide individually the best SSNR. In other words, it proves that given a low number of sensors, the sensors contained in the best subset are not individually the best sensors. This observation shows that the sensor subset should be consider directly as a whole and not as the aggregation of individual sensors in spite of the backward elimination procedure. Sensors should complement each other in the subset to enhance the P300 detection. This highlights the difference between two problems: evaluating the best sensor subsets for a P300-BCI, and estimating the best P300 projection on the scalp.

Finally, the sensor selection method is not based on the detection of the P300 but only on the estimation of the SSNR, thus avoiding different stages like classifiers training and cross validation. Spatial filtering is not only a necessary pre-processing 
Impact of spatial filters during sensor selection in a visual P300 Brain-Computer Interface16 step for signal detection, it is also a relevant pre-processing step for sensor selection.

This study allowed comparing different ways for analyzing the location of the P300 on the scalp. The different experiments have shown that it is possible to achieve relatively good performance with only four sensors, with an accuracy of about $90 \%$ with 10 epochs. For the different subsets of four sensors, which are personalized to each subject, two sensors are common to half of the subjects with $A S\left(P O_{9}, O_{1}\right)$. With $V S$, only $P_{8}$ is common to more than half of the subjects (Table 2). Some sensors are located on the occipital area, confirming previous works suggesting that occipital sites have also an important role $[19,15,20]$.

Although the P300 response has been discovered for over 40 years [21], its full understanding remains challenging and elusive [22]. The best locations for the sensors depend on the person and highlight both the complexity underlying the P300 process and the need of a personalized/adaptive P300-BCI with a limited number of sensors. Several studies in the literature have located the sources of the P300. Maeno et al. [23] point out the P300 sources on the bilateral occipito-temporal gyrus for visual stimuli as this area lies in the ventral pathway for vision. In [24], Yamaguchii et al. indicate that association cortex in the temporal-parietal junction is critical for generating the scalp-recorded target and novelty P300s, whereas dorsolateral frontal cortex contributes preferentially to novelty P300 generation. These sources are projected on the scalp and can explain the topographic maps presented in Figure 2. The inter-individual differences may be ascribed to the peculiar convolution of individual cortex with heterogeneous equivalent resulting dipole orientation [25]. For the P300 speller paradigm, the type of P300 detected corresponds mainly to the P3b, which is related to context updating operations and subsequent memory storage. As the P3b is generated via parieto-temporal cortical structures, it could explain the high rank of $P_{8}$ and $P_{7}$ for most of the subjects. Future 
Impact of spatial filters during sensor selection in a visual P300 Brain-Computer Interface17 works will deal with the sensor selection in relation 
Impact of spatial filters during sensor selection in a visual P300 Brain-Computer Interface18 Acknowledgment

The authors thank the anonymous reviewers for their pertinent comments which allow to drastically improve the quality of this paper.

This work has been supported by French National Research Agency (ANR) through

TecSan program (project RoBIK ANR-09-TECS-013) and through DEFIS program (project Co-Adapt ANR-09-EMER-002).

\section{References}

[1] B. Z. Allison, E. W. Wolpaw, and J. R. Wolpaw. Brain-computer interface systems: progress and prospects. Expert Review of Medical Devices, 4(4):463-474, 2007.

[2] N. Birbaumer and L. G. Cohen. Brain-computer interfaces: communication and restoration of movement in paralysis. Journal of Physiology-London, 579(3):621-636, 2007.

[3] C. Guger, G. Edlinger, W. Harkam, I. Niedermayer, and G. Pfurtscheller. How many people are able to operate an EEG-based brain-computer interface (BCI)? IEEE Trans. Neural Syst Rehabil Eng., 11(2):145-147, 2003.

[4] C. Guger, S. Daban, E. Sellers, C. Holznera, G. Krausza, R. Carabalonac, F. Gramaticac, and G. Edlinger. How many people are able to control a P300-based brain.computer interface (BCI)? Neuroscience Letters, 462:94-98, 2009.

[5] C. Sannelli, T. Dickhaus, S. Halder, E.-M. Hammer, K.-R. Müller, and B. Blankertz. On optimal channel configurations for SMR-based brain-computer interfaces. Brain Topogr, 23(2):186-193, 2010.

[6] E. I. Shih, A. H. Shoeb, and J. V. Guttag. Sensor selection for energy-efficient ambulatory medical monitoring. In Proc. of the 7th International Conference on Mobile Systems, Applications and Services, pages 347-358, 2009.

[7] U. Hoffmann, J. M. Vesin, K. Diserens, and T. Ebrahimi. An efficient P300-based brain-computer interface for disabled subjects. Journal of Neuroscience Methods, 167(1):115-125, 2008.

[8] T. N. Lal, M. Schroder, T. Hinterberger, J. Weston, M. Bogdan, N. Birbaumer, and B. Scholkopf. Support vector channel selection in BCI. IEEE Trans. Biomed. Engineering, 51(6):1003-1010, 2004 . 
Impact of spatial filters during sensor selection in a visual P300 Brain-Computer Interface19

[9] M. Schroder, T. N. Lal, T. Hinterberger, M. Bogdan, J. N. N. Jeremy Hill, N. Birbaumer, W. Rosenstiel, and B. Scholkopf. Robust EEG channel selection across subjects for braincomputer interfaces. EURASIP Journal on Applied Signal Processing, 19:3103-3112, 2005.

[10] E. Donchin, K. M. Spencer, and R. Wijesinghe. Assessing the speed of a P300-based braincomputer interface. IEEE Trans. Neural Sys. Rehab. Eng., 8(2):174-179, 2000.

[11] B. Rivet, A. Souloumiac, V. Attina, and G. Gibert. xDAWN algorithm to enhance evoked potentials: application to brain-computer interface. IEEE Trans Biomed Eng., 56(8):2035-43, 2009.

[12] E. Maby, G. Gibert, P.-E. Aguera, M. Perrin, O. Bertrand, and J. Mattout. The OpenViBE P300-Speller scenario: a thorough online evaluation. In Human Brain Mapping Conference, 2010.

[13] F. Sharbrough, G. Chatrian, and R. P. et al. Lesser. Guidelines for standard electrode position nomenclature. Bloomfield, IL: American EEG Society, 1990.

[14] L. Farwell and E. Donchin. Talking off the top of your head: toward a mental prosthesis utilizing event-related brain potentials. Electroencephalogr. Clin. Neurophysiol., 70:510-523, 1988.

[15] H. Cecotti and A. Gräser. Convolutional neural networks for P300 detection with application to brain-computer interfaces. IEEE Trans. Pattern Analysis and Machine Intelligence, 2010.

[16] A. Rakotomamonjy and V. Guigue. BCI competition iii : Dataset ii - ensemble of SVMs for BCI P300 speller. IEEE Trans. Biomedical Engineering, 55(3):1147-1154, 2008.

[17] D. J. C. MacKay. Bayesian interpolation. Neural Comput., 4(3):415-447, 1992.

[18] B. Rivet, A. Souloumiac, G. Gibert, V. Attina, and O. Bertrand. Sensor selection for P300 speller brain computer interface. In Proc. European Symposium on Artificial Neural Networks, pages 431-438, Bruges, Belgiunm, 2009.

[19] L. Bianchi, S. Sami, A. Hillebrand, I. P. Fawcett, L. R. Quitadamo, and S. Seri. Which physiological components are more suitable for visual ERP based brain-computer interface? a preliminary MEG/EEG study. Brain Topogr, 23(2):180-185, 2010.

[20] D. J. Krusienski, E. W. Sellers, D.J. McFarland, T. M. Vaughan, and J. R. Wolpaw. Toward enhanced P300 speller performance. Journal of Neuroscience Methods, 167:15-21, 2008.

[21] S. Sutton, M. Braren, J. Zubin, and E. R. John. Evoked potential correlates of stimulus uncertainty. Science, 150:1187-1188, 1965.

[22] J. Polich. Updating P300: An integrative theory of P3a and P3b. Clinical Neurophysiology, 
Impact of spatial filters during sensor selection in a visual P300 Brain-Computer Interface20 $118: 2128-2148,2007$.

[23] T. Maeno, A. Kaneko, K. Iramina, F. Eto, and S. Ueno. Source modeling of the P300 eventrelated response using magnetoencephalography and electroencephalography measurements. IEEE Trans on Magnetics, 39(5):3396-3398, 2003.

[24] S. Yamaguchii and R. T. Knight. Anterior and posterior association cortex contributions to the somatosensory P300. Journal of Neuroscience, 11(7):2039-2054, 1991.

[25] I. M. Tarkka and D. S. Stokic. Source localization of P300 from oddball, single stimulus, and omitted-stimulus paradigms. Brain Topography, 1(2):141-151, 1998. 